\title{
Microscopic Restoration of Proton-Neutron Mixed Symmetry in Weakly Collective Nuclei
}

\author{
J. D. Holt, $, 1,2$, N. Pietralla, ${ }^{1,3,4}$ J. W. Holt,${ }^{1}$ T. T. S. Kuo, ${ }^{1}$ and G. Rainovski ${ }^{1}$ \\ ${ }^{1}$ Department of Physics and Astronomy, SUNY, Stony Brook, NY 11794-3800, USA \\ ${ }^{2}$ TRIUMF, 4004 Wesbrook Mall, Vancouver, BC V6T 2A3, Canada \\ ${ }^{3}$ Institut für Kernphysik, Technische Universität Darmstadt, 64289 Darmstadt, Germany \\ ${ }^{4}$ Institut für Kernphysik, Universität zu Köln, 50937 Köln, Germany
}

(Dated: July 11, 2018)

\begin{abstract}
Starting from the microscopic low-momentum nucleon-nucleon interaction $V_{\text {low } k}$, we present the first systematic shell model study of magnetic moments and magnetic dipole transition strengths of the basic low-energy one-quadrupole phonon excitations in nearly-spherical nuclei. Studying in particular the even-even $N=52$ isotones from ${ }^{92} \mathrm{Zr}$ to ${ }^{100} \mathrm{Cd}$, we find the predicted evolution of the predominantly proton-neutron non-symmetric state reveals a restoration of collective proton-neutron mixed-symmetry structure near mid-shell. This provides the first explanation for the existence of pronounced collective mixed-symmetry structures in weakly-collective nuclei.

PACS numbers: 21.60.Cs, 21.30.-x,21.10.-k
\end{abstract}

Mesoscopic quantum systems such as Bose-Einstein condensates, superconductors, and quark-gluon systems are some of the most intensely studied in contemporary physics [1, 2]. Their dynamical properties are determined by the interplay and mutual balance of collective and single-particle degrees of freedom. In two fluid systems such as atomic nuclei, the presence of an isospin degree of freedom only serves to enhance this complexity. Of particular interest for understanding the physics of these systems is the microscopic origin of those excitations possessing collective two-fluid character. Collective quadrupole isovector excitations in the valence shell, socalled mixed-symmetry states (MSSs) [3], are the beststudied examples of this class of excitations. A special type of MSS, the $1^{+}$scissor mode, was predicted to exist [4] and discovered [5] in atomic nuclei. It is not surprising then, that analogous scissor-mode states have subsequently been found in other two-fluid quantum systems such as trapped Bose-Einstein condensed gases [6, 7], metallic clusters [8], and elliptical quantum dots [9].

Even though MSSs are a common feature of two-fluid quantum systems, atomic nuclei are still the primary laboratory in which our understanding of them can be shaped. In the interacting boson model of heavy nuclei (IBM-2) the definition of MSSs is formalized by the bosonic $F$-spin symmetry [3]. It arises predominantly from a collective coupling of proton and neutron sub-systems, and when the proton/neutron (pn) valence spaces are large enough, strong coupling can arise between them. Naturally then, the best examples of pnsymmetric and MSSs would be expected at mid shells. However, pronounced MSSs have recently also been observed in weakly collective nuclei especially in $N=52$ isotones. Multiphonon structures of MSSs are observed in the nucleus ${ }^{94} \mathrm{Mo}$ [10, 11, 12], in neighboring nuclei

*Electronic address: jholt@triumf.ca
13, 14, 15], and recently the first MSSs in an oddmass nearly-spherical nucleus were identified in ${ }^{93} \mathrm{Nb}[16]$. Even though the experimental properties of MSSs in this region have been well described within the framework of the IBM-2 [17, 18], the nuclear shell model (SM) [14, 19], and the quasiparticle-phonon model [20], the question of how these states arise and evolve has not yet been answered.

In this Letter, we provide the first microscopic foundation for the formation and evolution of the fundamental MSS of vibrational nuclei, the one-quadrupole phonon $2_{1, \mathrm{~ms}}^{+}$state, from SM calculations using the lowmomentum nucleon-nucleon (NN) interaction $V_{\text {low } k}$ [21]. $V_{\text {low } k}$ defines a new class of $\mathrm{NN}$ interaction with a variable momentum cutoff (or resolution scale) that reproduces low-energy two-nucleon observables. For $N=52$ isotones from the $Z=40$ to the $Z=50$ shell closures, we investigate the structure of the two SM one-phonon $2^{+}$states (labeled $2_{I}^{+}$and $2_{I I}^{+}$), where the $2_{I I}^{+}$state is connected to the $2_{I}^{+}$state by a strong $M 1$ transition. The $2_{I}^{+}$and $2_{I I}^{+} \mathrm{SM}$ states, of course, correspond to the experimentally-observed one-phonon symmetric $2_{1}^{+}$and mixed symmetric $2_{1, \mathrm{~ms}}^{+}$states, respectively. The evolution of these states is seen by examining two of the most important observables: $B\left(M 1 ; 2_{I I}^{+} \rightarrow 2_{I}^{+}\right)$values and magnetic moments. Our calculations indicate that the quantity driving this evolution is the orbital proton contribution to the $M 1$ transitions. Moreover, the main cause underlying the formation of MSSs in these weakly collective nuclei is the approximate energy degeneracy in the proton and the neutron one-phonon quadrupole excitations. This mechanism can be considered as a $m i$ croscopic restoration of proton-neutron symmetry. Because of the universal nature of MS states, these studies should serve as qualitative predictions of MS formation and structural evolution in other nuclear regions, and give parallel insight into analogous structures in general mesoscopic quantum systems. 


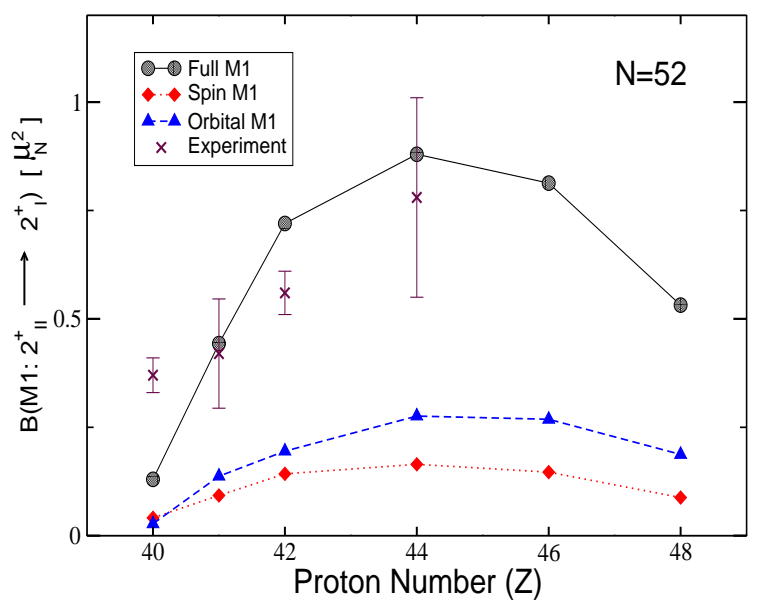

FIG. 1: (Color Online) Evolution of the total, orbital, and spin $B\left(M 1 ; 2_{I I}^{+} \rightarrow 2_{I}^{+}\right)$values for $N=52$ isotones. The experimental values are from [12, 13, 15, 16].

Our microscopic shell model calculations are based on the low-momentum NN interaction $V_{\text {low } k}$. Although various NN potentials have been developed which reproduce the NN data up to momenta of $\sim 2 \mathrm{fm}^{-1}$ [22], they differ in their treatment of the high-momentum modes, which are known to complicate many-body calculations. Using the renormalization group, we start from one of the high-precision interactions and integrate out the highmomentum components above a cutoff $\Lambda$ such that the physics below this cutoff is preserved. It has been shown that as $\Lambda$ is lowered to $2.1 \mathrm{fm}^{-1}$, the $V_{\text {low } k}$ interactions flow to a result largely independent of the input interaction 21, 23]. Furthermore, $V_{\text {low } k}$ is energy independent and thus suitable for SM calculations in any nuclear region 24]. For the present work we use a $V_{\text {low } k}$ interaction derived from the CD-Bonn [25] potential with a cutoff $\Lambda=2.1 \mathrm{fm}^{-1}$. Using the two-body matrix elements of $V_{\text {low } k}$, we then derive a SM effective interaction $V_{\text {eff }}$ based on the folded diagram methods detailed in [26]. Through this process we include the effects of core polarization to second order, which has been shown to be a reasonable approximation to all order effects in the absence of many-body interactions [27]. Finally, we note that $V_{\text {low } k}$ with a fixed cutoff has been successfully used in recent nuclear structure studies [28].

The present calculations were carried out with the Oxbash code [29] using the effective interaction described above. ${ }^{88} \mathrm{Sr}$ was used as the inert core and the protonneutron model space taken to be: $\pi:\left[2 p_{1 / 2}, 1 g_{9 / 2}\right]$ and $\nu:\left[1 g_{7 / 2}, 2 d_{5 / 2}, 2 d_{3 / 2}, 3 s_{1 / 2}, 1 h_{11 / 2}\right]$, where the single particle energies were taken from the experimental values in ${ }^{89} \mathrm{Sr}$ and ${ }^{89} \mathrm{Y}$. Following this procedure we have obtained a good description of the extensive experimental data on MSSs for ${ }^{92} \mathrm{Zr}$ and ${ }^{94} \mathrm{Mo}[30]$ and predicted the properties of MSSs in the odd-mass nearly spherical nucleus ${ }^{93} \mathrm{Nb}[16,30]$. It is debatable whether or not the ${ }^{88} \mathrm{Sr}$ core is suitable for such calculations due to the close

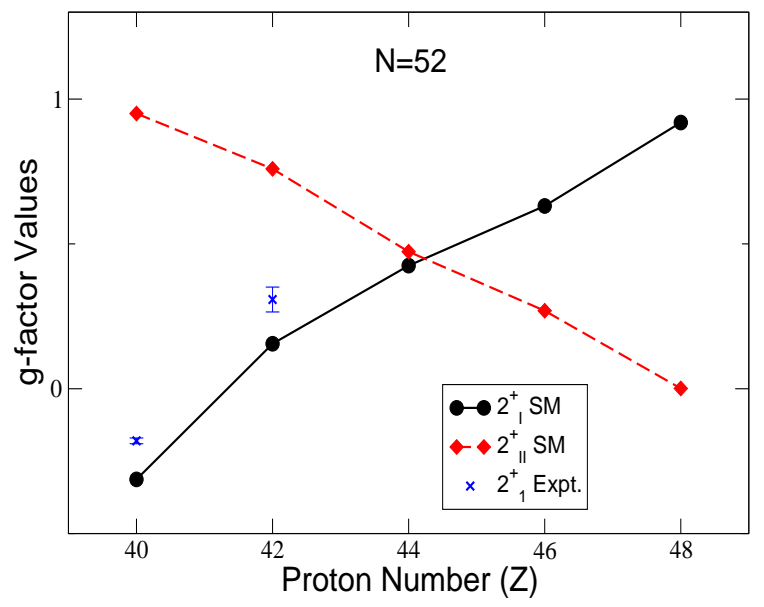

FIG. 2: (Color Online) Predicted evolution of the $g$-factors for the $2_{I}^{+}$and $2_{I I}^{+}$states across the series of $\mathrm{N}=52$ isotones. The experimental values are from [31, 32].

proximity of both the $2 p_{3 / 2}$ and the $1 f_{5 / 2}$ proton orbits. We should emphasize, however, that our goal is not a highly accurate reproduction of experimental data, but to understand global features of MSSs in terms of this simplified model space.

The key experimental signature for MSSs is a strong $M 1$ transition from the $2_{1}^{+}$to the $2_{1, \mathrm{~ms}}^{+}$state, due to the isovector character of the $M 1$ operator. The strength and fragmentation of this transition is an indicator of the purity of the MSSs. Bare orbital gyromagnetic factors $g_{\pi}^{l}=1 \mu_{N}, g_{\nu}^{l}=0$, with empirical (but not fine-tuned to fit the data) spin factors $g_{\pi}^{s}=3.18 \mu_{N}$, and $g_{\nu}^{s}=-2.18 \mu_{N}$ were used for calculating our SM $B\left(M 1 ; 2_{I I}^{+} \rightarrow 2_{I}^{+}\right)$values shown in Fig. 1. They show a pronounced parabolic behavior, maximized at mid-subshell. Also plotted in Fig. 1 are the spin and orbital contributions, both exhibiting an approximately parabolic shape.

The increase in $M 1$ strength from ${ }^{92} \mathrm{Zr}$ to ${ }^{96} \mathrm{Ru}$ is in good qualitative agreement with the data, though the SM calculations underpredict the value in ${ }^{92} \mathrm{Zr}$ and somewhat overpredict it in ${ }^{94}$ Mo. In Fig. 1 for comparison purposes, we have also included the average value for the $M 1$ transitions from the mixed-symmetry doublet states, $\frac{5}{2}^{-} \frac{3}{2}^{-}$ms , to the like- $J$ one-phonon states in ${ }^{93} \mathrm{Nb}[16]$; it is clearly in general agreement with the overall trend. For ${ }^{98} \mathrm{Pd}$ and ${ }^{100} \mathrm{Cd}$, however, a decrease in $M 1$ strength is predicted.

Figure 2 shows the predicted evolution of the $g$-factors of the $2_{I}^{+}$and $2_{I I}^{+}$states. As proton number increases the $2_{I}^{+} g$-factors increase almost linearly while the $2_{I I}^{+}$ $g$-factors decrease linearly with approximately the same absolute slope. As a result they cross at mid-shell. The calculated $g$-factors in ${ }^{96} \mathrm{Ru}$ for the $2_{I}^{+}$and $2_{I I}^{+}$states are close (0.42 and 0.48, respectively) and not far from the $Z / A=0.458$ value expected for a fully collective state. The negative $g$-factor $(-0.31)$ predicted for the $2_{I}^{+}$ state in ${ }^{92} \mathrm{Zr}$ is in qualitative agreement with experiment 


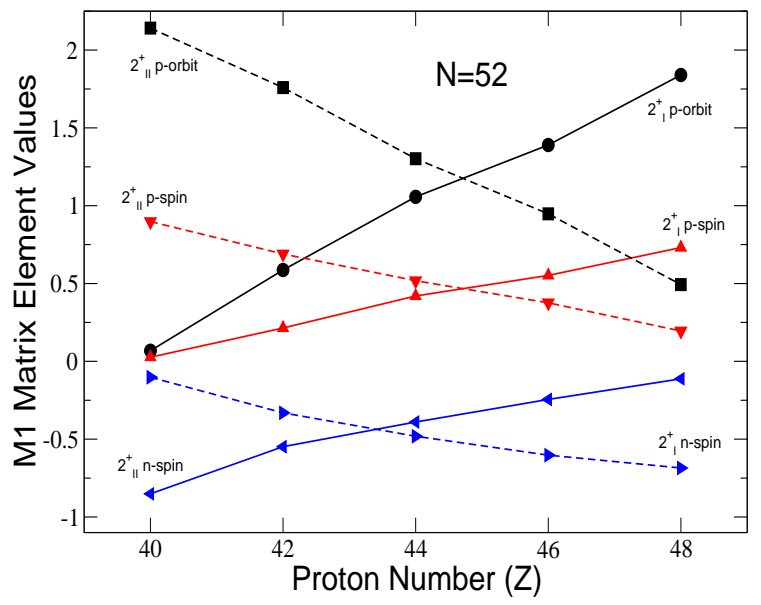

FIG. 3: (Color Online) Calculated proton/neutronorbital/spin matrix elements contributing to the $g$-factors.

[14, 31], indicating significant neutron character of this state. At $Z=40$ the $2_{I}^{+}$state of ${ }^{92} \mathrm{Zr}$ is expected to be primarily neutron in character. In drastic contrast, we predict a large positive $g$-factor for the $2_{I I}^{+}$state in ${ }^{92} \mathrm{Zr}$, which suggests a dominant proton character, from the $J=2$ coupling of protons in the $\pi\left(g_{9 / 2}^{2}\right)$ orbit. The unbalanced proton-neutron content of the ${ }^{92} \mathrm{Zr} 2_{I}^{+}$and $2_{I I}^{+}$states implies severe breaking of $F$-spin symmetry, not a surprise for such a weakly collective system. This $F$-spin breaking is reflected in the small $M 1$ transition strength (see Fig. 1). The changes in the $M 1$ strengths and the $g$-factors for the $2_{I}^{+}$and $2_{I I}^{+}$states from ${ }^{92} \mathrm{Zr}$ to ${ }^{100} \mathrm{Cd}$ isotopes consistently show the evolution of pnsymmetry character of the states: the purity of the MS character of the $2_{I I}^{+}$state peaks in the mid-shell region before waning at the approach of the $\mathrm{Z}=50$ shell closure.

The $\mathrm{p} / \mathrm{n}$-orbit/spin contributions to the $M 1$ matrix elements, $\left\langle 2_{i}^{+}\left\|g_{\pi, \nu}^{l, s} \cdot(M 1)_{\pi, \nu}^{l, s}\right\| 2_{i}^{+}\right\rangle_{(i=I, I I)}$ are shown in Fig. 3. Considering first the $2_{I}^{+}$states, we see that in ${ }^{92} \mathrm{Zr}$, the neutron-spin $\left(g_{\nu}^{s} \cdot M 1_{\nu}^{s}\right)$ component dominates, resulting in the negative $g$-factor. In ${ }^{94} \mathrm{Mo}$, however, the $M 1_{\pi}^{l}$ contribution has risen while the $M 1_{\nu}^{s}$ has declined, yielding a small and slightly positive $g$-factor. For the rest of the isotones, the $M 1_{\pi}^{l}$ component continues increasing, while the others become more negligible. For the $2_{I I}^{+}$states, the opposite situation is seen. The $M 1_{\pi}^{l}$ term remains the driving force behind the evolution of the $g$-factor, starting at a large, positive value in ${ }^{92} \mathrm{Zr}$. It remains dominant until near $Z=50$ where it has decreased to near zero while the $M 1_{\nu}^{s}$ element has become negative enough to influence the $g$-factor. The observed prominence of the orbital matrix element further confirms the collective nature of these excitations.

The calculated SM wavefunctions are generally complicated, but as discussed in [19], MS structure is evident in the dominant components. Table@gives the dominant amplitudes for the normalized $2_{I}^{+}$and $2_{I I}^{+}$wavefunctions. With the exception of the $2_{I}^{+}$state in ${ }^{92} \mathrm{Zr}$, the signifi-
TABLE I: Amplitudes of the dominant SM configurations contributing to the $J^{\pi}=2^{+}$one-phonon wavefunctions.

\begin{tabular}{cccc}
\hline \hline $\begin{array}{c}\text { Nucleus } \\
{ }^{92} \mathrm{Zr}\end{array}$ & $\pi\left(g_{9 / 2}^{2}\right)_{0} \nu\left(d_{5 / 2}^{2}\right)_{2}$ & 0.462 & -0.129 \\
& $\pi\left(g_{9 / 2}^{2}\right)_{2} \nu\left(d_{5 / 2}^{2}\right)_{0}$ & 0.078 & 0.725 \\
${ }^{94} \mathrm{Mo}$ & $\pi\left(p_{1 / 2}^{2} g_{9 / 2}^{2}\right)_{0} \nu\left(d_{5 / 2}^{2}\right)_{2}$ & 0.682 & -0.461 \\
& $\pi\left(p_{1 / 2}^{2} g_{9 / 2}^{2}\right)_{2} \nu\left(d_{5 / 2}^{2}\right)_{0}$ & 0.426 & 0.652 \\
${ }^{96} \mathrm{Ru}$ & $\pi\left(p_{1 / 2}^{2} g_{9 / 2}^{4}\right)_{0} \nu\left(d_{5 / 2}^{2}\right)_{2}$ & 0.586 & -0.584 \\
& $\pi\left(p_{1 / 2}^{2} g_{9 / 2}^{4}\right)_{2} \nu\left(d_{5 / 2}^{2}\right)_{0}$ & 0.512 & 0.548 \\
${ }^{98} \mathrm{Pd}$ & $\pi\left(p_{1 / 2}^{2} g_{9 / 2}^{6}\right)_{0} \nu\left(d_{5 / 2}^{2}\right)_{2}$ & 0.510 & -0.681 \\
& $\pi\left(p_{1 / 2}^{2} g_{9 / 2}^{6}\right)_{2} \nu\left(d_{5 / 2}^{2}\right)_{0}$ & 0.576 & 0.448 \\
${ }^{100} \mathrm{Cd}$ & $\pi\left(p_{1 / 2}^{2} g_{9 / 2}^{8}\right)_{0} \nu\left(d_{5 / 2}^{2}\right)_{2}$ & 0.376 & -0.787 \\
& $\pi\left(p_{1 / 2}^{2} g_{9 / 2}^{8}\right)_{2} \nu\left(d_{5 / 2}^{2}\right)_{0}$ & 0.638 & 0.305 \\
\hline \hline
\end{tabular}

cant contributions come from the $\pi\left(p_{1 / 2}^{2} g_{9 / 2}^{k}\right)_{0} \nu\left(d_{5 / 2}^{2}\right)_{2}$ and $\pi\left(p_{1 / 2}^{2} g_{9 / 2}^{k}\right)_{2} \nu\left(d_{5 / 2}^{2}\right)_{0}$ configurations, where $k=2$ for ${ }^{94} \mathrm{Mo}, k=8$ for ${ }^{100} \mathrm{Cd}$, and the protons are jointly coupled to either $J=0$ or $J=2$. For ${ }^{92} \mathrm{Zr}$ we have only listed the $\pi\left(g_{9 / 2}^{2}\right) \nu\left(d_{5 / 2}^{2}\right)$ configuration since the two protons in $\pi\left(p_{1 / 2}^{2}\right)$ cannot couple to $J=2$. These components only represent a part of the full wavefunctions, but the percentage is typically on the order of $60 \%$ to $70 \%$. From Table 1 we see that the dominant parts of the $2_{I}^{+}$ and $2_{I I}^{+}$wavefunctions generally exhibit a pn-symmetric structure. Even with these drastically truncated wavefunctions, the $2_{I}^{+}$and $2_{I I}^{+}$are formed by the same dominant components and are approximately orthogonal: we find $\left\langle 2_{I}^{+} \mid 2_{I I}^{+}\right\rangle<0.1$ in every case. The evolution of the $M 1$ strength and the $g$-factors is apparent - at the low end of the isotone chain, the $2_{I}^{+}$state is predominantly neutron in character and the $2_{I I}^{+}$state is largely proton. In ${ }^{94}$ Mo already both components are more equally important. For the $2_{I}^{+}$state, there is a linear increase in proton character and decrease in neutron character across the shell with the opposite behavior seen in the $2_{I I}^{+}$states. At mid-shell in ${ }^{96} \mathrm{Ru}$ both states have almost equal absolute amplitude of proton and neutron excitations forming the fully symmetric and mixed symmetry one-quadrupole phonon states.

These approximated wavefunctions and their $M 1$ matrix elements can be considered in the seniority scheme. The proton and neutron contributions to the predominant seniority-two $(\nu=2)$ parts of the one-quadrupole phonon wavefunctions in this sequence of isotones can be related to the fractional filling $f=(Z-40) / 10$ of the $\pi\left(g_{9 / 2}\right)$ orbital with protons. The wavefunctions can be approximated as

$$
\left|2_{I}^{+}\right\rangle \approx \sqrt{f}\left|2_{\pi}^{+}\right\rangle+\sqrt{1-f}\left|2_{\nu}^{+}\right\rangle
$$




$$
\left|2_{I I}^{+}\right\rangle \approx \sqrt{1-f}\left|2_{\pi}^{+}\right\rangle-\sqrt{f}\left|2_{\nu}^{+}\right\rangle
$$

where the $\left|2_{\pi(\nu)}^{+}\right\rangle$represents a SM configuration in which the protons (neutrons) are coupled to $2^{+}$and the neutrons (protons) are coupled to $0^{+}$. To the extent that this two-state decomposition represents a large percentage of the total wavefunctions, we can deduce

$$
\begin{aligned}
g\left(2_{I}^{+}\right) & =\sqrt{\frac{2 \pi}{45}}\left[\mu_{\nu}+f\left(\mu_{\pi}-\mu_{\nu}\right)\right] \\
g\left(2_{I I}^{+}\right) & =\sqrt{\frac{2 \pi}{45}}\left[\mu_{\pi}-f\left(\mu_{\pi}-\mu_{\nu}\right)\right] \\
B\left(M 1 ; 2_{I I}^{+} \rightarrow 2_{I}^{+}\right) & =\frac{1}{5} f(1-f)\left(\mu_{\pi}-\mu_{\nu}\right)^{2}
\end{aligned}
$$

with $\mu_{\pi(\nu)}=\left\langle 2_{\pi(\nu)}^{+}\|M 1\| 2_{\pi(\nu)}^{+}\right\rangle$being the diagonal $M 1$ matrix elements of the $\nu=2$ proton (neutron) $2^{+}$ configuration. Note that $\mu_{\nu}<0$ is a constant for the $N=$ 52 isotones and, within the seniority scheme, the matrix element $\mu_{\pi}>0$ is independent of the filling of the proton orbital and, hence, a constant as well. It immediately follows that the $g$-factor of the $2_{I}^{+}$state increases linearly with the filling of the $\pi\left(g_{9 / 2}\right)$ orbital while the $g$-factor of the $2_{I I}^{+}$state linearly drops, instead, with the same absolute slope. In contrast to this, the $M 1$ transition strength is proportional to the factor $f(1-f)$ and exhibits a parabolic collective behavior over the $\pi\left(g_{9 / 2}\right)$ shell with a maximum at mid-shell. The size of the $B\left(M 1 ; 2_{I I}^{+} \rightarrow\right.$ $\left.2_{I}^{+}\right)$value is proportional to the quadratic slope of the $g$ factors. This relation is quantitatively $\left(\mu_{\pi}-\mu_{\nu} \approx 4.2 \mu_{N}\right)$ in good agreement even with the full calculations.

Finally, the shell model results are qualitatively at variance with the $F$-spin limit of the IBM-2. With effective boson $g$-factors $g_{\rho} \equiv \sqrt{2 \pi / 45} \mu_{\rho}$ the predictions of the $F$-spin limit of the U(5) dynamical symmetry limit are

$$
\begin{aligned}
g\left(2_{1}^{+}\right) & =\sqrt{\frac{2 \pi}{45}}\left[\mu_{\nu}+\frac{N_{\pi}}{N}\left(\mu_{\pi}-\mu_{\nu}\right)\right] \\
g\left(2_{1, \mathrm{~ms}}^{+}\right) & =\sqrt{\frac{2 \pi}{45}}\left[\mu_{\pi}-\frac{N_{\pi}}{N}\left(\mu_{\pi}-\mu_{\nu}\right)\right] \\
B\left(M 1 ; 2_{1, \mathrm{~ms}}^{+} \rightarrow 2_{1}^{+}\right) & =\frac{1}{5} \frac{N_{\pi}}{N}\left(1-\frac{N_{\pi}}{N}\right)\left(\mu_{\pi}-\mu_{\nu}\right)^{2}
\end{aligned}
$$

which is formally identical to Eqs. (224) with the replacement $f \rightarrow N_{\pi} / N$ and $2_{I}^{+}=2_{1}^{+}, 2_{I I}^{+}=2_{1, \mathrm{~ms}}^{+}$. However, due to the convention of counting bosons as half of the number of valence particles or holes outside the nearest closed shell, the boson number fraction $N_{\pi} / N$ does not correspond to the fractional filling $f$ of the proton shell but is instead for $N_{\nu}=1$ approximately equal to the fractional half-filling. The $F$-spin limit of the IBM-2 together with the conventional counting of bosons would lead to local extrema for the $g$-factors of the one-phonon $2^{+}$states at mid-shell [maximum for $g\left(2_{1}^{+}\right)$and minimum for $\left.g\left(2_{1, \mathrm{~ms}}^{+}\right)\right]$and to a reduction of the $B\left(M 1 ; 2_{1, \mathrm{~ms}}^{+} \rightarrow 2_{1}^{+}\right)$ value from ${ }^{94} \mathrm{Mo}$ to ${ }^{96} \mathrm{Ru}$ in contradiction to the data.
The discrepancy in predicted evolution between the $F$-spin limit of the IBM-2 and the SM calculation using $V_{\text {low } k}$ originates from the breaking of $F$-spin symmetry and from its specific restoration near mid-shell in the shell model. The smooth change in the proton-neutron character of the $2_{I}^{+}$and $2_{I I}^{+}$states, evident from Table 1 . can be attributed to the variation of energies of the onephonon proton and neutron configurations with filling of the $\pi\left(g_{9 / 2}\right)$ orbital. This can be seen in the data for the semi-closed shell nuclei in the vicinity of the $N=52$ isotones. The $2_{1}^{+}$energy of the even $N=50$ isotones is indicative of the energy of the $2_{\pi}^{+}$excitation. It drops slightly from $1509 \mathrm{keV}$ in ${ }^{92} \mathrm{Mo}$ to $1394 \mathrm{keV}$ in ${ }^{98} \mathrm{Cd}$. On the other hand, the energies of $2_{1}^{+}$states in the ${ }^{92} \mathrm{Zr}$ and ${ }^{102} \mathrm{Sn}$ are indicative of the energy of the $2_{\nu}^{+}$excitation. The data increase from $934 \mathrm{keV}$ in ${ }^{92} \mathrm{Zr}$ to $1472 \mathrm{keV}$ in ${ }^{102} \mathrm{Sn}$. The difference in energy of these basic $2_{\pi(\nu)}^{+}$excitations translates into the IBM-2 framework in a difference of proton and neutron $d$-boson energies that cause a breaking of $F$-spin symmetry. Due to the low collectivity near shell closure, this breaking cannot be restored by strong enough pn-coupling. As a result, $F$-spin breaking is most pronounced in ${ }^{92} \mathrm{Zr}$ [15] and must be expected for ${ }^{100} \mathrm{Cd}$, too. The energy crossing of the $2_{\pi}^{+}$and $2_{\nu}^{+}$ configurations near mid-shell, where the collectivity also peaks (see Table I), causes a specific restoration of $F$ spin symmetry, yielding there the largest $B(M 1)$ values and almost equal $g$-factors. This process is microscopic in origin and differs from the usual $F$-spin symmetry generation due to the collective pn-coupling in the framework of the IBM-2.

In summary we have used the low-momentum $\mathrm{NN}$ interaction $V_{\text {low } k}$ to provide a microscopic description of the evolution of MSSs and magnetic dipole collectivity studying the $N=52$ isotones as a particular example. The predicted observables reveal a new specific restoration of proton-neutron symmetry which originates from the energy degeneracy of basic proton and neutron excitations. This process offers for the first time an explanation for the existence of pronounced $2_{1, \mathrm{~ms}}^{+}$structures in weakly collective nuclei and might be observable in other two-fluid quantum systems.

\section{Acknowledgments}

We would like to thank B. A. Brown for his invaluable assistance with the Oxbash code. Support from the US Department of Energy under contract DE-FG0288ER40388 and the Natural Sciences and Engineering Research Council of Canada (NSERC) is gratefully acknowledged. TRIUMF receives federal funding via a contribution agreement through the National Research Council of Canada. 
[1] W. Ketterle, Rev. Mod. Phys. 74, 1131 (2002).

[2] V. L. Ginzburg, Rev. Mod. Phys. 76, 981 (2004).

[3] F. Iachello, Phys. Rev. Lett. 53, 1427 (1984).

[4] N. Lo Iudice and F. Palumbo, Phys. Rev. Lett. 41, 1532 (1978).

[5] D. Bohle, A. Richter, W. Steffen, A. E. L. Dieperink, N. Lo Iudice, F. Palumbo, and O. Scholten, Phys. Lett. B137, 27 (1984).

[6] D. Guéry-Odelin and S. Stringari, Phys. Rev. Lett. 83, 4452 (1999).

[7] O. M. Maragó, S. A. Hopkins, J. Arlt, E. Hodby, G. Hechenblaikner, and C. J. Foot, Phys. Rev. Lett. 84, 2056 (2000).

[8] V. O. Nesterenko, W. Kleinig, F. F. de Souza Cruz, and N. Lo Iudice, Phys. Rev. Lett. 83, 57 (1999).

[9] L. Serra, A. Puente, and E. Lipparini, Phys. Rev. B 60, R13 966 (1999).

[10] N. Pietralla, C. Fransen, D. Belic, P. von Brentano, C. Friessner, U. Kneissl, A. Linnemann, A. Nord, N H. Pitz, T. Otsuka, I. Schneider, V. Werner, and I. Wiedenhöver, Phys. Rev. Lett. 83, 1303 (1999).

[11] N. Pietralla, C. Fransen, P. von Brentano, A. Dewald, A. Fitzler, C. Friessner, and J. Gableske, Phys. Rev. Lett. 84, 3775 (2000).

[12] C. Fransen, N. Pietralla, Z. Ammar, D. Bandyopadhyay, N. Boukharouba, P. von Brentano, A. Dewald, J. Gableske, A. Gade, J. Jolie, U. Kneissl, S. R. Lesher, A. F. Lisetskiy, M. T. McEllistrem, M. Merrick, H. H. Pitz, N. Warr, V. Werner, and S. W. Yates, Phys. Rev. C 67, 024307 (2003).

[13] N. Pietralla, C. J. Barton III, R. Krücken, C. W. Beausang, M. A. Caprio, R. F. Casten, J. R. Cooper, A. A. Hecht, H. Newman, J. R. Novak, and N. V. Zamfir, Phys. Rev. C 64, 031301(R) (2001).

[14] V. Werner, D. Belic, P. von Brentano, C. Fransen, A. Gade, H. von Garrel, J. Jolie, U. Kneissl, C. Kohstall, A. Linnemann, A. F. Lisetskiy, N. Pietralla, H. H. Pitz, M. Scheck, K.-H. Speidel, F. Stedile, and S. W. Yates, Phys. Lett. B550, 140 (2002).

[15] C. Fransen, V. Werner, D. Bandyopadhyay, N. Boukharouba, S. R. Lesher, M. T. McEllitrem, J. Jolie, N. Pietralla, P. von Brentano, and S. W. Yates, Phys.
Rev. C 71, 054304 (2005).

[16] J. N. Orce, J. D. Holt, A. Linnemann, C. J. McKay, S. R. Lesher, C. Fransen, J. W. Holt, A. Kumar, N. Warr, V. Werner, J. Jolie, T. T. S. Kuo, M. T. McEllistrem, N. Pietralla, and S. W. Yates, Phys. Rev. Lett. 97, 062504 (2006).

[17] F. Iachello and A. Arima, The Interacting Boson Model, Oxford University Press, 1990.

[18] P. van Isacker, K. Heyde, J. Jolie, and A Sevrin, Ann. Phys. (N. Y.) 171, 253 (1986).

[19] A. F. Lisetskiy, N. Pietralla, C. Fransen, R. V. Jolos, and P. von Brentano, Nucl. Phys. A677, 100 (2000).

[20] N. Lo Iudice and Ch. Stoyanov Phys. Rev. C 62, 047302 (2000); 65, 064304 (2002); 69, 044312 (2004); 73, 037305 (2006).

[21] S. K. Bogner, T. T. S. Kuo, and A. Schwenk, Phys. Rep. 386, 1 (2003).

[22] R. Machleidt and I. Slaus, J. Phys G 27 R69 (2001).

[23] S. K. Bogner, T. T. S. Kuo, A. Schwenk, D. R. Entem, and R. Machleidt, Phys. Lett. B576, 265 (2003).

[24] S. K. Bogner, T. T. S. Kuo, L. Coraggio, A. Covello, and N. Itaco, Phys. Rev. C 65, 051301(R) (2002).

[25] R. Machleidt, Phys. Rev. C 63, 024001 (2001).

[26] T. T. S. Kuo and E. Osnes, Lecture Notes in Physics, Vol. 364, Springer-Verlag, New York (1990).

[27] J. D. Holt, J. W. Holt, T. T. S. Kuo, G. E. Brown, and S. K. Bogner, Phys. Rev. C 72, 041304(R) (2005).

[28] L. Coraggio, A. Covello, A. Gargano, and N. Itaco, Phys. Rev. C 70, 034310 (2004); 72, 057302 (2005); 73, 031302(R) (2006).

[29] B. A. Brown, A. Etchegoyen, N. S. Godwin, W. D. M. Rae, W. A. Richter, W. E. Ormand, E. K. Warburton, J. S. Winfield, L. Zhao, and C. H. Zimmerman, MSU-NSCL report number 1289 .

[30] J. D. Holt et al., in preparation.

[31] J. Jakob, N. Benczer-Koller, J. Holden, G. Kumbartzki, T. J. Mertzimekis, K.-H. Speidel, C. W. Beausang, and R. Krücken, Phys. Lett. B468, 13 (1999).

[32] P. F. Mantica, A. E. Stuchbery, D. E. Groth, J. I. Prisciandaro, and M. P. Robinson, Phys. Rev. C 63, 034312 (2001). 